\title{
Utilização de serviços de urgência e emergência por complicações agudas da hipertensão e/ou diabetes
}

\author{
Use of urgency and emergency services for acute hypertension and / or diabetes complications \\ Uso de servicios de urgencia y emergencia para complicaciones agudas de hipertensión a y / o \\ diabetes
}

Patrícia Chatalov Ferreira ${ }^{1}$ (D) Elen Ferraz Teston ${ }^{2}$ (1) Verônica Francisqueti Marquete ${ }^{1}$ (1) Rubia Mariana de Souza Santos ${ }^{1}$ (1) Robson Marcelo Rossi ${ }^{1}$ (1) Sonia Silva Marcon ${ }^{1}$ (1)

1. Universidade Estadual de Maringá.

Maringá, PR, Brasil.

2. Universidade Federal de Mato Grosso do Sul. Campo Grande, MS, Brasil.
Autor correspondente:

Patrícia Chatalov Ferreira.

E-mail: pattychatalovf@gmail.com.

Recebido em 01/02/2021.

Aprovado em 05/07/2021.

\section{RESUMO}

Objetivo: analisar a utilização de serviços de urgência e emergência por indivíduos com complicações agudas de Hipertensão Arterial e/ou Diabetes Mellitus. Método: estudo retrospectivo realizado nos três serviços públicos de pronto-atendimento em município do Sul do Brasil. Os dados foram coletados mediante consulta aos prontuários eletrônicos das pessoas que compareceram duas ou mais vezes no período de janeiro de 2018 a fevereiro de 2020. Na análise foi utilizado Regressão Logística e georreferenciamento dos endereços residenciais. Resultados: das 1.125 pessoas, $72,7 \%$ procuraram os serviços por Hipertensão; 18,1\% por Diabetes; e 7,3\%, por ambas as condições. A maioria não possuía a condição crônica registrada na Atenção Primária e residia próximo aos serviços de pronto-atendimento. Tiveram mais chances de três ou mais comparecimentos os usuários que procuraram concomitantemente as Unidades de Pronto atendimento do Hospital Universitário e as municipais por ambas as condições, com menor escolaridade e sem companheiro. Conclusão e implicações para a prática: informações sobre a utilização dos serviços de urgência e emergência e das pessoas que os procuram por complicações agudas da Hipertensão e/ou Diabetes podem subsidiar a formulação de políticas públicas e a proposição de estratégias mais efetivas na identificação, acompanhamento e busca ativa de pessoas com condições crônicas.

Palavras-chave: Hipertensão; Diabetes Mellitus; Serviços Médicos de Emergência; Atenção Primária à Saúde; Avaliação em Saúde.

\begin{abstract}
Objective: to analyze the use of urgency and emergency services by individuals with acute complications of Hypertension and/ or Diabetes Mellitus. Method: a retrospective study conducted in three public emergency care services in a city in southern Brazil. Data was collected by consulting the electronic medical records of people who attended two or more times during the period from January 2018 to February 2020. Logistic Regression and geo-referencing of residential addresses were used in the analysis. Results: of the 1,125 people, $72.7 \%$ sought the services for hypertension; $18.1 \%$ for diabetes; and $7.3 \%$ for both conditions. Most did not have the chronic condition registered in Primary Care and lived near the emergency services. Users who sought the Emergency Care Units of the University Hospital and the municipal ones concomitantly, for both conditions, with less education, and without a partner had a greater chance of three or more attendances. Conclusion and implications for practice: information on the use of urgency and emergency services and of people who seek them for acute complications of Hypertension and/or Diabetes can subsidize the formulation of public policies and the proposition of more effective strategies in the identification, follow-up and active search for people with chronic conditions.
\end{abstract}

Keywords: Hypertension; Diabetes Mellitus; Emergency Medical Services; Primary Health Care; Health Evaluation.

\section{RESUMEN}

Objetivo: analizar el uso de los servicios de urgencia y emergencia por personas con complicaciones agudas de Hipertensión Arterial y / o Diabetes Mellitus. Método: estudio retrospectivo realizado en los tres servicios públicos de emergencia de un municipio del sur de Brasil. Los datos se recogieron consultando las historias clínicas electrónicas de las personas que acudieron dos o más veces entre enero de 2018 y febrero de 2020. En el análisis se utilizó la Regresión Logística y la georreferenciación de domicilios. Resultados: de las 1125 personas, el 72,7\% buscó servicios por hipertensión, el 18,1\% por diabetes mellitus y el 7,3\% por ambas condiciones. La mayoría no tenía la enfermedad crónica registrada en Atención Primaria y vivía cerca de los servicios de emergencia. Los usuarios que acudieron de forma concurrente a las Unidades Hospitalarias de Urgencias del Hospital Universitario y las municipales, por ambas condiciones, con menor escolaridad y sin pareja tenían más probabilidades de tener tres o más apariciones. Conclusión e implicaciones para la práctica: las informaciones sobre el uso de los servicios de urgencia y emergencia y las personas que los buscan por complicaciones agudas de Hipertensión Arterial y / o Diabetes Mellitus pueden apoyar la formulación de políticas públicas y la propuesta de estrategias más efectivas en la identificación seguimiento y búsqueda activa de personas con enfermedades crónicas.

Palabras clave: Hipertensión; Diabetes Mellitus; Servicios Médicos de Urgencia; Atención Primaria de Salud; Evaluación en Salud. 


\section{INTRODUÇÃO}

A Hipertensão Arterial Sistêmica (HAS) e o Diabetes Mellitus (DM) configuram as principais causas de morte e invalidez em todo o mundo ${ }^{1,2}$. Estima-se que até 2050 seis países de renda média, entre eles o Brasil, apresentarão mudanças demográficas importantes, com aumento considerável do número de adultos que necessitarão de tratamento para doenças crônicas ${ }^{3}$. No Brasil, a prevalência estimada da HAS corrigida pelos dados autorreferidos da Pesquisa Nacional de Saúde em 2013, foi de 14,5\% .

Relatório que estimou o ônus das doenças crônicas, em 195 países e territórios, de 1990 a 2016, constatou que para o DM houve um aumento de $117,9 \%$ no número de anos vividos com incapacidade ${ }^{5}$. Em 2018 houve 1.829 .779 internações por causas associadas à HAS, ao DM e à obesidade no Sistema Único de Saúde (SUS), o que corresponde a, aproximadamente, $16 \%$ do total de internações hospitalares e a um custo total de $\mathrm{R} \$ 3,84$ bilhões, sendo que a HAS sozinha foi responsável por $59 \%$ deste custo (mais de $\mathrm{R} \$ 2$ bilhões por ano), e o diabetes por $30 \%{ }^{1}$.

Ainda no Brasil, $28 \%$ do total de óbitos ocorrem por doenças cardiovasculares, acarretando um aumento significativo de $17 \%$ nos custos, que só no ano de 2015 foi de $R \$ 37,1$ bilhões (US\$ 9.6 bilhões), incluindo os gastos estimados por morte prematura, custos diretos com internações e perdas de produtividade relacionados a essas condições ${ }^{6}$.

Destarte, a elevada prevalência de HAS e DM na população, também associada à elevada frequência de suas complicações agudas e crônicas, faz com que essas condições constituam importante problema de saúde coletiva no Brasil ${ }^{7}, 8$. As complicações decorrentes do descontrole dessas enfermidades geram impactos negativos tanto na qualidade de vida dos indivíduos acometidos quanto no sistema de saúde ${ }^{9}$. Porém, a implementação de medidas de prevenção e tratamento adequado dessas condições representam um grande desafio para os profissionais e gestores da área de saúde?.

Embora a caracterização dos usuários frequentes nos serviços de Urgência e Emergência proporcionem insights valiosos tanto para pesquisadores quanto para formuladores de políticas públicas ${ }^{10}$, constata-se a escassez de estudos nacionais que abordem a procura por esses serviços pelos usuários com complicações agudas da HAS e/ou DM. Esse fato aponta a relevância do tema e a necessária discussão sobre medidas e estratégias voltadas para o controle das condições crônicas nos diferentes pontos da rede de assistência, inclusive nas unidades de Pronto Atendimento (PA).

Considerando-se que o conhecimento sobre o perfil das entradas nos serviços de urgência e emergência por essas causas e das pessoas que os procuram, além de sinalizar lacunas existentes na Rede de Atenção (RA), também poderá contribuir para o desenvolvimento e aperfeiçoamento de estratégias e políticas públicas de assistência voltadas a esses usuários, definiu-se, como objetivo deste estudo, analisar a utilização de serviços de urgência e emergência por indivíduos com complicações agudas de Hipertensão Arterial e/ou Diabetes Mellitus.

\section{MÉTODO}

Estudo retrospectivo que utilizou como fonte dos dados os prontuários eletrônicos da Secretaria Municipal de Saúde (SMS) e do Hospital Universitário de um município da região Sul do Brasil, localizado a Noroeste do estado do Paraná, com estimativa populacional de 423.666 habitantes e $70,9 \%$ de cobertura pela Estratégia Saúde da Família. A Rede de Atenção é composta por 34 Unidades Básicas de Saúde (UBS), duas Unidades de Pronto Atendimento municipal (UPA), Policlínica, Ambulatórios, Consórcio Público Intermunicipal de Saúde (CISAMUSEP), Hospital Universitário (HU), Hospital Municipal e instituições privadas credenciadas ao SUS.

Em 2014, o município iniciou a implementação de um processo de avaliação para conceder títulos de reconhecimento às equipes com base nas propostas do Programa de Qualificação da Atenção Primária à Saúde (APSUS) e do Programa Nacional de Melhoria do Acesso e da Qualidade da Atenção Básica (PMAQ-AB). Atualmente, duas UBS possuem o selo Ouro; uma possui o Prata; 12 possuem o selo bronze; e 19 UBS ainda não foram avaliadas. Todas as UBS certificadas iniciaram a implementação do Modelo de Atenção às Condições Crônicas (MACC) com estratificação dos usuários crônicos, encaminhamento e acompanhamento para a atenção especializada.

A população do estudo foi composta pelos registros de entrada dos indivíduos nos três serviços públicos de Pronto Atendimento do município (UPA Zona Sul, UPA Zona Norte e do Hospital Universitário) durante um período de 26 meses: janeiro de 2018 a fevereiro de 2020 . Os dados foram coletados no período de dezembro de 2019 a março de 2020, mediante consulta aos sistemas de cada serviço, com a aplicação de filtros sequencias: ano e CID-10, ressaltando-se que na UPA Zona Norte, o prontuário eletrônico foi implantando em 2019.

Os critérios de inclusão estabelecidos foram: residir no município e ter duas ou mais entradas motivadas por causas relacionadas à HAS e/ou DM no período em estudo, considerando-se que uma única entrada poderia ser um evento isolado, motivado por questões emocionais e que não configuraria descontrole da condição. Já os critérios de exclusão foram: óbito, dados incompletos e idade inferior a 18 anos.

Os CID relacionados à HAS foram: I10, I11, I11.0, I11.9, I12, I12.0, I12.9, I13, I13.0, I13.1, I13.2, I13.9, G45, G45.8, I64, I21, I21.0, I21.1, I21.2, I21.3, I21.4, e I21.9, O11, I20, I20.8, I20.9. Por sua vez, os CID referentes à Diabetes Mellitus foram: E10 a E10.9, E11 a E11.9, E13 a E13.9, E14 a E14.9, N08.3, O24.0 a O24.3, E16.0 a E16.2, G59.0, G63.2, H28.0, H36.0, M14.2 e R73.9.

As entradas levantadas foram organizadas em planilha do Microsoft Office Excel $2020 \circledast$ contendo: número de prontuário, nome do usuário, serviço de saúde procurado, data e hora do atendimento e o respectivo CID. As pessoas com duas ou mais entradas foram identificadas e, posteriormente, foi acessado o prontuário da (SMS) para coleta de dados cadastrais: data de nascimento, Unidade Básica de Saúde de referência, endereço domiciliar, situação familiar, escolaridade, cor da pele, estado conjugal, religião e registro da condição "HAS" e/ou "DM" 
cadastrada no prontuário eletrônico da (SMS), ao utilizar o filtro "condição referida cadastrada".

Constatou-se que 7.632 pessoas foram responsáveis pelo total de 10.649 entradas nos serviços de Urgência e Emergência por HAS e/ou DM. Destas, 1.316 tiveram duas ou mais entradas, e 191 foram excluídas (134 não residiam no município, 48 foram a óbito, quatro tinham menos de 18 anos e cinco tinham dados incompletos), sendo elegíveis para o estudo 1.125 pessoas.

Utilizou-se o programa $\mathrm{R}^{11}$ para análise dos dados, por meio do Modelo de Regressão Logística Múltipla ${ }^{12-14}$, empregando o método Forward-Backward Stepwise, em que as variáveis explicativas são inseridas ou extraídas do modelo de acordo com ordem do valor de significância, permitindo determinar o melhor ajuste por meio do critério de Akaike (AIC).

A variável desfecho foi o número de entradas: duas e três ou mais. As variáveis explicativas foram: sexo, cor da pele, idade, escolaridade, estado civil, condição de saúde cadastrada no prontuário eletrônico, serviço procurado, motivo da entrada e UBS de referência certificada.

A medida de associação entre as variáveis desfecho e explicativas foi a OddsRatio (OR), e respectivos intervalos com $95 \%$ de confiança, considerando-se como desfecho a variável aleatória com distribuição binomial, Y: número de entradas \{0: até 2 e 1: 3 ou mais\}. Para a verificação da qualidade do modelo de regressão final ajustado utilizou-se o teste de Hosmer e Lemeshow $(H-L)^{14}$, além da obtenção de medida de área sob a curva ROC (AUC) $)^{15}$ e verificação gráfica do comportamento dos resíduos via envelope simulado binomial ${ }^{16}$.
No que concerne à distribuição espacial, foram identificadas as medidas de coordenadas (latitude e longitude) através do Google Maps dos endereços das 1.125 pessoas, utilizando-se a base cartográfica disponibilizada no site do município e do Instituto de Brasileiro de Geografia e Estatística (IBGE). A compilação dos resultados e a produção cartográfica foram realizadas com auxílio dos programas BatchgeoPro, ArcGis 10.4.1 e CorelDraw X8. O primeiro foi utilizado para a geocodificação do banco de dados (endereços). $O$ segundo é uma ferramenta SIG (Sistema de Informação Geográfica) que permite a visualização da densidade dos pontos. $\mathrm{E}$ o último, executou o layout final do mapa. A densidade de pontos permite avaliar o padrão de dispersão de uma variável sobre determinada superfície ${ }^{17}$. Mediante o emprego dessa ferramenta, o programa gera um dado matricial que apresenta a estimativa da densidade de pontos que foram identificados pelo point density.

O estudo foi desenvolvido em consonância com o preconizado nas Resoluções 466/2012 e 510/2016 do Conselho Nacional de Saúde, e o projeto foi aprovado pelo Comitê de Ética em Pesquisas com Seres Humanos da instituição signatária (CAAE 26346019.1.0000.0104).

\section{RESULTADOS}

As 1.125 pessoas incluídas no estudo foram responsáveis por 3.061 entradas nos serviços de Urgência e Emergência, sendo que $818(72,7 \%)$ tiveram como diagnóstico registrado na ficha de atendimento a HAS e/ou complicações; 204 (18,1\%), DM e/ou complicações; e 103 (7,3\%), ambas as condições. Na Tabela 1

Tabela 1. Perfil das entradas e da cobertura da Atenção Primária de pessoas que buscaram os serviços de urgência e emergência em decorrência da Hipertensão e/ou Diabetes e/ou suas complicações em um município do Sul do Brasil.

\begin{tabular}{|c|c|c|c|c|c|c|}
\hline \multirow{3}{*}{ Variáveis } & \multicolumn{6}{|c|}{ Número de entradas: } \\
\hline & \multicolumn{2}{|c|}{ Duas entradas } & \multicolumn{2}{|c|}{$\geq$ Três entradas } & \multicolumn{2}{|c|}{ TOTAL } \\
\hline & $\mathrm{N}$ & $\%$ & $\mathrm{~N}$ & $\%$ & $\mathrm{~N}$ & $\%$ \\
\hline \multicolumn{7}{|l|}{ Entrada por } \\
\hline HAS e/ou complicações & 534 & 47,5 & 284 & 25,2 & 818 & 72,7 \\
\hline DM e/ou complicações & 131 & 11,6 & 73 & 6,5 & 204 & 18,1 \\
\hline HAS e DM e/ou complicações & 47 & 4,2 & 56 & 5,0 & 103 & 9,2 \\
\hline \multicolumn{7}{|l|}{ Serviço que procurou } \\
\hline UPA & 690 & 61,3 & 337 & 30,0 & 1027 & 91,3 \\
\hline Pronto atendimento hospitalar & 6 & 0,5 & 10 & 0,9 & 16 & 1,4 \\
\hline Ambos os serviços & 16 & 1,4 & 66 & 5,9 & 82 & 7,3 \\
\hline \multicolumn{7}{|l|}{ Condição referida cadastrada } \\
\hline Nenhuma & 402 & 35,7 & 220 & 19,6 & 622 & 55,3 \\
\hline Hipertensão & 162 & 14,4 & 99 & 8,8 & 261 & 23,2 \\
\hline Diabetes & 37 & 3,3 & 21 & 1,9 & 58 & 5,2 \\
\hline Hipertensão e Diabetes & 111 & 9,9 & 73 & 6,5 & 184 & 16,4 \\
\hline \multicolumn{7}{|l|}{ UBS certificada } \\
\hline Sim & 320 & 28,4 & 194 & 17,2 & 514 & 45,7 \\
\hline Não & 392 & 34,8 & 219 & 19,5 & 611 & 54,3 \\
\hline
\end{tabular}


são apresentadas características relacionadas aos atendimentos e a cobertura dessas pessoas na Atenção Primária. Chama a atenção o fato de que mais da metade $(55,3 \%)$ dessas pessoas não teve condição referida registrada no prontuário eletrônico da UBS de referência, embora quase metade delas pertencesse à área de abrangência de UBS certificada pelo APSUS (45,7\%).

As características sociodemográficas das pessoas deste estudo constam na Tabela 2, demonstrando-se que quase metade dos indivíduos que procuraram os serviços de pronto atendimento tinha menos de 60 anos ( $47,7 \%)$, e a maioria possuía baixa escolaridade $(71,4 \%)$.

Por sua vez, na Tabela 3 são apresentadas as variáveis associadas ao maior número de entradas. Constata-se que maiores chances de três ou mais entradas foram observadas nas pessoas que procuraram apenas o pronto atendimento do Hospital Universitário (PA-HU) ou este em concomitância com uma das duas UPA, motivados por queixas relacionadas a complicações de ambas as condições (HAS e DM), incluindose a informação de que não possuíam companheiro e tinham baixa escolaridade.
Na Figura 1, a seguir, apresenta-se a distribuição espacial dos endereços de residência das pessoas que procuraram os serviços de urgência e emergência.

Observa-se que dos serviços de Urgência e Emergência - representados pela letra "H" - , a UPA Zona Sul (no lado inferior direito) está localizada na região com maior densidade populacional, seguida da UPA Zona Norte (canto superior direito) e PA-HU (canto superior esquerdo).

Também se constata semelhança na distribuição dos endereços nos três mapas: independente da condição que motivou a procura, maior utilização ocorre por pessoas que residem próximo a estes serviços. Contudo, o primeiro mapa mostra que as pessoas com HAS e/ou complicações que procuraram esses serviços eram oriundas de todas as regiões do município, inclusive da área rural. Ademais, mostra que não existe diferença de o indivíduo residir ou não em área de abrangência de UBS certificada. Porém, chama a atenção o fato de que, nos três mapas, poucas pessoas residentes na área de abrangência de uma UBS certificada em Bronze, localizada na região nor-noroeste, procuraram os serviços de urgência e emergência no período em estudo.

Tabela 2. Características sociodemográficas de pessoas que buscaram os serviços de urgência e emergência em decorrência da Hipertensão e/ou Diabetes e/ou suas complicações em um município do Sul do Brasil.

\begin{tabular}{|c|c|c|c|c|c|c|}
\hline \multirow{3}{*}{ Variáveis } & \multicolumn{6}{|c|}{ Número de entradas: } \\
\hline & \multicolumn{2}{|c|}{ Duas entradas } & \multicolumn{2}{|c|}{$\geq$ Três entradas } & \multicolumn{2}{|c|}{ TOTAL } \\
\hline & $\mathrm{n}$ & $\%$ & $\mathrm{~N}$ & $\%$ & $\mathrm{~N}$ & $\%$ \\
\hline \multicolumn{7}{|l|}{ Sexo $(n=1125)$} \\
\hline Masculino & 308 & 27,4 & 179 & 15,9 & 487 & 43,3 \\
\hline Feminino & 404 & 35,9 & 234 & 20,8 & 638 & 56,7 \\
\hline \multicolumn{7}{|l|}{ Cor da pele $(n=1110)$} \\
\hline Branca & 484 & 43,6 & 295 & 26,6 & 779 & 70,2 \\
\hline Não branca & 219 & 19,7 & 112 & 10,1 & 331 & 29,8 \\
\hline \multicolumn{7}{|l|}{ Idade ( $n=1125)$} \\
\hline $18+\dashv 39$ & 83 & 7,4 & 41 & 3,6 & 124 & 11,0 \\
\hline $40+\dashv 59$ & 275 & 24,4 & 138 & 12,3 & 413 & 36,7 \\
\hline $60+$ & 354 & 31,5 & 234 & 20,8 & 588 & 52,3 \\
\hline \multicolumn{7}{|l|}{ Escolaridade ( $n=1063$ ) } \\
\hline Sem estudo & 28 & 2,6 & 27 & 2,5 & 55 & 5,2 \\
\hline $1-9$ anos & 433 & 40,7 & 271 & 25,5 & 704 & 66,2 \\
\hline$\geq 10$ anos & 213 & 20,0 & 91 & 8,6 & 304 & 28,6 \\
\hline \multicolumn{7}{|c|}{ Situação Familiar ( $n=1103$ ) } \\
\hline Mora com alguém & 658 & 59,7 & 390 & 35,4 & 1048 & 95,0 \\
\hline Mora sozinho & 38 & 3,4 & 17 & 1,5 & 55 & 5,0 \\
\hline \multicolumn{7}{|l|}{ Estado civil ( $n=1058$ ) } \\
\hline Com companheiro & 441 & 41,7 & 243 & 23,0 & 684 & 64,7 \\
\hline Sem companheiro & 222 & 21,0 & 152 & 14,4 & 374 & 35,3 \\
\hline
\end{tabular}


Tabela 3. Regressão logística entre a variável desfecho (número de entradas) e as variáveis explicativas para pessoas que buscaram os serviços de Urgência e Emergência por HAS e/ou DM e/ou complicações em um município do Sul do Brasil, 2020.

\begin{tabular}{lcc}
\hline \multicolumn{1}{c}{ Variáveis } & OR (IC 95\%) & valor- $p$ \\
\hline Serviço que deu entrada & Baseline & - \\
UPA & $3,8(1,3-12,7)$ & 0,017 \\
\hline PA-HU & $11,0(6,0-21,9)$ & $<0,001$ \\
\hline Ambos os serviços & Baseline & - \\
\hline Motivo da entrada & - & $0,275^{\text {ns }}$ \\
\hline Entrada por HAS/complicações & $2,4(1,6-3,8)$ & $<0,001$ \\
\hline Entrada por DM/complicações & - & $0,158^{\text {ns }}$ \\
\hline Entrada por ambas as condições & $1,4(1,0-1,8)$ & 0,035 \\
\hline Situação Familiar & & 0,029 \\
\hline Estado Civil & $2,0(1,1-3,9)$ & 0,048 \\
\hline Escolaridade & $1,4(0,6-1,1)$ & - \\
\hline Não estudou & baseline & $0,117^{\text {ns }}$ \\
\hline 1 a 9 anos de estudo & - & \\
\hline 10 ou mais de estudo & & \\
\hline Cor da pele & & \\
\hline
\end{tabular}

H-L: $p=0,76 ; A \cup C=0,67$ Ns: Não significativa.

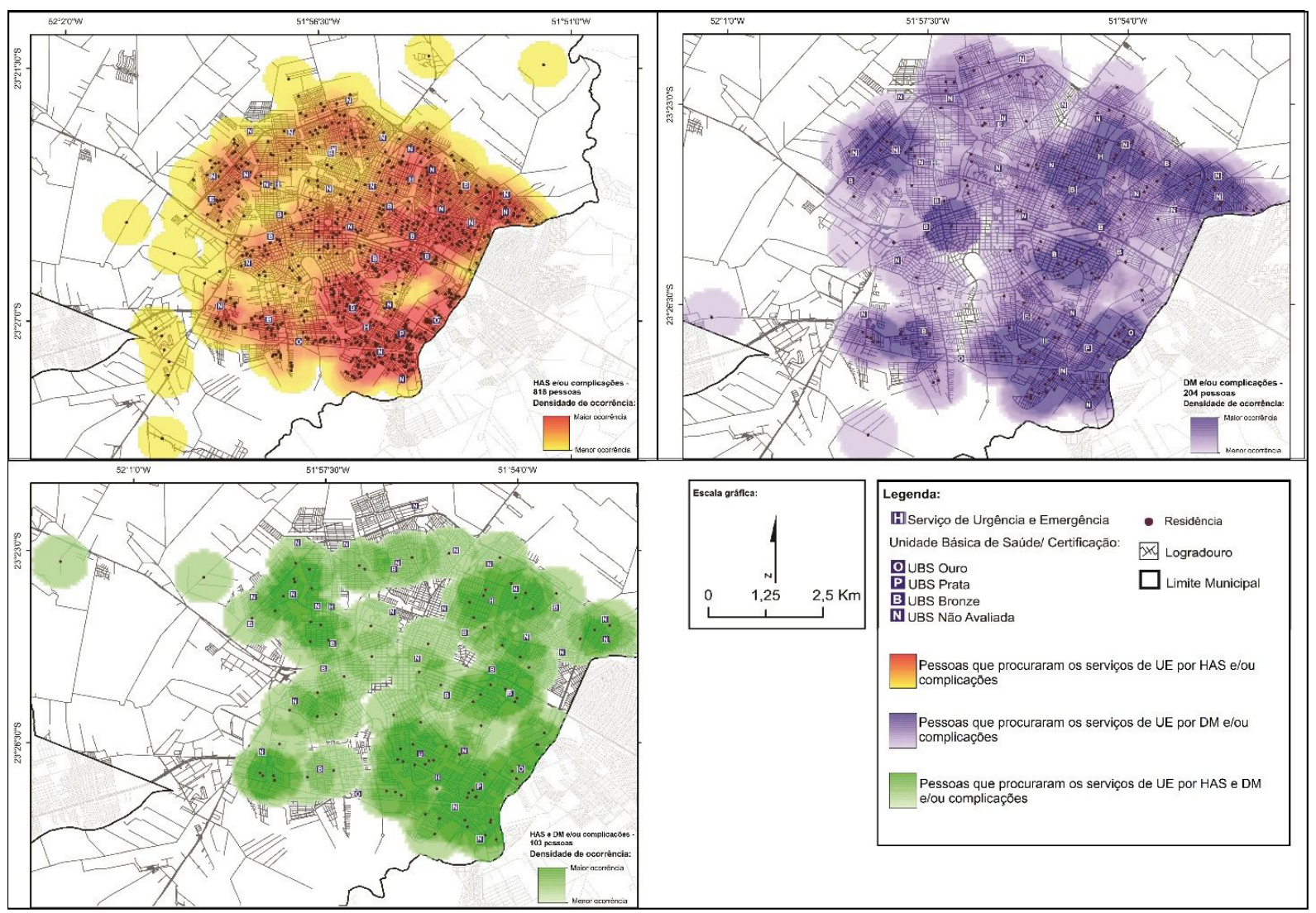

Figura 1. Georreferenciamento dos endereços residenciais de pessoas que buscaram os serviços de urgência e emergência em decorrência da Hipertensão e/ou Diabetes e/ou suas complicações no período de janeiro de 2018 a fevereiro de 2020 , em município do Noroeste do estado do Paraná, 2020. Fonte: Dados da pesquisa (2020). 


\section{DISCUSSÃO}

O número de pessoas que procuraram os serviços de Pronto Atendimento por duas ou mais vezes, em decorrência de complicações agudas da HAS e/ou DM, é considerado alto, pois, essas doenças integram o rol de Condições Sensíveis a Atenção Primária (CSAP), considerando-se que $80 \%$ dos casos podem ser prevenidos e tratados nesse nível assistencial ${ }^{18}$. Outros estudos também dizem ser frequente a presença de pessoas com crise hipertensiva, descontrole glicêmico e complicações agudas nas unidades de urgência e emergência ${ }^{2,19-21}$.

Destarte, algumas características desses serviços, por exemplo porta aberta nas 24 horas do dia, possibilidade de atendimento médico e realização de exames sem necessidade de agendamento prévio e boa resolutividade para condições agudas, concorrem para torná-los mais atrativos, acessíveis e muito utilizados pela população. Essa procura também é influenciada, em parte, pela hegemonia do modelo biomédico nos serviços de saúde e não adoção de ações/comportamentos preventivos por parte dos usuários em geral ${ }^{22}$.

Estudo realizado em um município do estado de Minas Gerais, por exemplo, constatou que os participantes com HAS e DM, quando apresentavam sintomas de descontrole não procuravam o serviço de saúde, mas se automedicavam até conquistar a estabilização do quadro ${ }^{7}$. Desse modo, a busca por serviços de pronto atendimento pode sinalizar uma dificuldade em alcançar o controle da doença. Logo, as pessoas que procuram os serviços de Urgência e Emergência por essas causas precisam sempre ser orientadas e encaminhadas para o adequado acompanhamento na Atenção Primária à Saúde (APS), a fim de limitar a evolução da doença e o surgimento de (novas) complicações, contribuindo para o seu melhor prognóstico e sobrevida.

$\mathrm{O}$ fato de as pessoas que procuraram o PA do $\mathrm{HU}$ ou ambos os serviços apresentarem mais chance de buscar serviços de emergência três ou mais vezes pode decorrer de dois fatores: da tomada de ciência sobre o agravamento da condição e medo das consequências por não tratar a doença7; da percepção maior quanto a sua resolutividade, provavelmente em função da existência de maiores recursos diagnósticos e de maior diversidade de especialistas atuando no serviço.

A percepção de uma assistência mais rápida e completa, motivada pela oferta de exames diagnósticos, também foi apontada por usuários do serviço de emergência de um hospital de grande porte no Rio Grande do Sul. Outros motivos referidos para a busca do serviço foram: exacerbação de doenças crônicas, facilidade de acesso, percepção de maior resolutividade, oferta de tecnologia diagnóstica, vínculo com os profissionais e retornos agendados $^{19}$.

Para além dessas questões, foi evidenciado que um número significativo de indivíduos que procuraram os serviços de pronto atendimento por descontrole glicêmico ou dos níveis pressóricos não tinha diagnóstico de hipertensão e/ou Diabetes Mellitus cadastrado no prontuário da UBS. Isto pode caracterizar ausência de diagnóstico prévio e, consequentemente, de gerenciamento de seu manejo, o que pode ter influência na procura recorrente desses serviços por complicações/manifestações agudas.

Em um estudo de base populacional, em que os autores avaliaram os fatores associados ao acompanhamento de adultos com HAS e/ou DM pelas equipes de Saúde da Família em um município do Norte do Paraná, foi constatado que $40,3 \%$ dos participantes não tinham registro da condição nas unidades básicas. Além disso, o acompanhamento pelas equipes não atendia ao padrão assistencial estabelecido para os grupos, pois até as pessoas estratificadas como alto risco cardiovascular não receberam o número de visitas domiciliares, consultas de enfermagem e médicas propostos pelo Ministério da Saúde ${ }^{23}$.

A ausência ou falha no registro do diagnóstico de HAS e/ou DM no prontuário eletrônico da Rede de Atenção do município, não necessariamente significa que essas pessoas não estejam sendo assistidas na APS. Porém, pode afetar os tributos essenciais da APS, como a coordenação da atenção e o acompanhamento efetivo da condição ${ }^{24}$, além de impossibilitar a identificação da real magnitude do problema pelos gestores, impactando o desenvolvimento de políticas públicas e distribuição de recursos e serviços condizentes com o contexto existente.

Os resultados do presente estudo, portanto, mostram, em certa medida, que as condições crônicas continuam sendo enfrentadas na mesma lógica das condições agudas, ou seja, a partir da demanda espontânea, com enfoque curativista e uso de tecnologias destinadas a responder aos momentos de agudização, resultando na procura por assistência nos serviços de urgência e emergência ${ }^{25}$. Destarte, sem o contínuo e adequado acompanhamento na rede de atenção à saúde, os eventos agudos tornam-se mais frequentes e, num processo cíclico, a utilização dos serviços de urgência e emergência também ${ }^{19}$.

O ideal é que, sendo tratada a agudização da condição crônica, seja garantida a continuidade da assistência na APS e, quando necessário, na atenção especializada e terciária ${ }^{25}$. $\mathrm{O}$ adequado acompanhamento do usuário na APS e especializada pode reduzir o número de atendimentos por descontroles pressóricos e/ou glicêmicos não só nos serviços de urgência e emergência, mas também em outros pontos da rede de assistência ${ }^{21}$.

Essas questões apontam a importância da busca ativa e de um sistema de referência e contrarreferência que permita a continuidade do cuidado e o acesso às ações de prevenção de complicações oferecidas nas unidades de saúde. É provável que o uso sistematizado de um programa de rastreamento favoreça a identificação das pessoas que procuram os serviços de Urgência e Emergência por complicações agudas da HAS e/ ou DM e que desconhecem sua condição de saúde ou que não são adequadamente acompanhadas na APS².

As pessoas que buscaram os PA com queixas relacionadas a ambas as condições (HAS e DM) apresentarem 43\% mais chances de três ou mais entradas em serviço de urgência e emergência, o que reafirma que a associação de ambas tende a agravar a condição de saúde e, por conseguinte, desencadear mais episódios de agudização. 
Para o efetivo controle da condição de saúde é primordial que a pessoa acometida entenda a importância do manejo adequado e da adoção cotidiana de ações de autocuidado ${ }^{7} \mathrm{e}$ que haja melhorias nos serviços ofertados na Atenção Primária, após ter sido constatado controle insatisfatório da HAS e do DM nesse nível de atenção ${ }^{23,26}$. Nesse sentido, pesquisa realizada no Sudoeste da Bahia, com 352 usuários com DM, constatou que a maioria dos entrevistados apresentava descontrole glicêmico e falha no controle pressórico e metabólico, o que apresentou associação com receber menos visitas dos agentes comunitários e não possuir outras comorbidades ${ }^{27}$.

Em relação à UBS de referência, ao contrário do observado, esperava-se que pessoas residentes em áreas de abrangência daquelas certificadas procurassem menos os serviços de urgência e emergência por descompensação dos níveis pressóricos e glicêmicos, pois os programas de qualificação e certificação da APS avaliam muitos indicadores, inclusive alguns relacionados ao acompanhamento e controle da HAS e DM. Pesquisa que analisou a produção das equipes da Estratégia Saúde da Família antes e após a implantação do PMAQ-AB em Florianópolis, Santa Catarina, também não evidenciou diferença nos resultados obtidos pelas equipes aderentes e não aderentes ${ }^{24}$.

Referente às características dos participantes, chama a atenção o fato de quase metade deles ter menos de 60 anos, o que pode estar relacionado à dificuldade de acesso às UBS, devido à coincidência no horário de funcionamento dessas Unidades e o trabalho ${ }^{19,28-30}$ e às características dos serviços de Urgência e Emergência - porta aberta 24 horas por dia, e recursos diagnósticos disponíveis no mesmo dia do atendimento, não sendo necessário faltar no trabalho ${ }^{19}$. Além disso, também pode constituir um indicativo de que as complicações agudas da HAS e DM estão surgindo em uma etapa da vida cada vez mais precoce.

As pessoas sem companheiro(a) apresentaram 38\% de chance de ter três ou mais entradas se comparadas com as com companheiro(a), o que permite inferir que elas constituem fonte de apoio para o enfrentamento dessas condições no dia a dia. Essa dedução é reforçada por estudo que aponta a importância do papel da mulher no núcleo familiar, a qual, além de atuar como cuidadora, favorecendo maior controle da doença, também incentiva que o cônjuge procure serviços de saúde diante de intercorrências ${ }^{22}$.

Outras pessoas do núcleo familiar mais próximo também podem apoiar o autocuidado e ajudar no manejo da condição crônica. Desse modo, é essencial que a equipe de saúde esteja capacitada para realizar atividades educativas com foco na promoção da saúde e prevenção de agravos, e que essas ações sejam dirigidas às pessoas com condições crônicas e seus familiares, que favoreçam a troca de saberes e valorizem a criação de vínculo, a singularidade de cada indivíduo e seu contexto sociocultural ${ }^{30}$

Pessoas com menor escolaridade apresentaram maiores chances de buscar três ou mais vezes os serviços de pronto atendimento, o que, de certo modo, corrobora achados que apontam que a baixa escolaridade relaciona-se à deficiência na adoção de ações de autocuidado ${ }^{27}$ e a maior procura por serviços de saúde em geral ${ }^{22,31}$. Nessa mesma direção, estudos revelam que a baixa escolaridade se associa à menor Literacia em Saúde, o que, por sua vez, afeta o entendimento sobre a condição saúde/doença, resultando em maior dificuldade na adesão às ações de autocuidado, tanto no âmbito preventivo quanto curativo ${ }^{32,33}$.

O georreferenciamento mostrou que a maioria das pessoas que buscaram os serviços de pronto atendimento reside em sua proximidade, demonstrando que usuários residentes nas regiões Nordeste, Sudeste, Sudoeste e Noroeste possuem maior acessibilidade aos serviços de urgência do município. Inclusive, em algumas regiões não houve procura dos seus residentes pelos serviços de emergência em estudo, o que permite inferir que, naquelas regiões, não há pessoas acometidas por essas condições ou, se acometidas, elas conseguem manter o controle ou utilizam serviços de saúde privados.

Em Salvador, Bahia, estudo com usuários de um serviço hospitalar de emergência também identificou associação de sua utilização com a proximidade geográfica ${ }^{20}$. Porém, na China, autores constataram que a maior procura por esses serviços não ocorre somente devido à proximidade, mas porque os usuários confiam mais nesses serviços do que nas clínicas ou UBS e isto, além de sobrecarregar esses serviços, gera ineficiência em toda a Rede ${ }^{29}$. Frente a isso, destaca-se a necessidade de ações que resultem no fortalecimento da territorialização e da busca ativa, porque o conhecimento dos indivíduos adscritos possibilita a vinculação dos deles aos serviços de saúde e, consequentemente, um acompanhamento melhor e longitudinal de suas condições de saúde-doença.

Os resultados do presente estudo elucidam a importância da utilização de ferramentas, como os sistemas de informações locais, para o diagnóstico situacional de condições que afetam de modo prevalente a saúde da população. Cabe ressaltar que os dados obtidos oferecem subsídios para o planejamento de intervenções direcionadas à organização dos serviços localizados nos diferentes pontos da Rede de Atenção à Saúde.

Ressalta-se que a distribuição espacial dos endereços de residência dos usuários e suas características contribuem para a formulação de hipóteses que explicam a magnitude do fenômeno pesquisado, por exemplo, os fatores associados aos recorrentes quadros de descompensação pressórica e glicêmica. A análise de padrões de pontos (PPA) tem sido amplamente utilizada para investigar os padrões de distribuição espacial global ou local de agravos pontuais ${ }^{17}$, além de contribuir para que haja equidade, otimização e alocação adequada dos recursos de saúde, de modo a mitigar as disparidades dos serviços ofertados ${ }^{29}$.

Informações estatísticas precisas e fidedignas são essenciais para a gestão no SUS, porque permitem conhecer e avaliar a real conjuntura de cada território, contribuindo para a elaboração de estratégias mais efetivas, melhor cobertura da população e equidade dos serviços disponibilizados ${ }^{34}$. Logo, conhecer as características predominantes das pessoas que procuram 
serviços de urgência e emergência por condições sensíveis à APS - como HAS e/ou DM - e ter ciência se essas condições estão cadastradas e se são ou não reconhecidas nesse nível de assistência é essencial, à medida que aponta lacunas a serem exploradas e consideradas pela gestão, ao se ponderar que esse grupo pode estar desassistido.

Os resultados encontrados permitem corroborar a recomendação feita pelo estudo realizado na Tanzânia, o qual apontou a importância de reconhecer que os serviços de urgência e emergência constituem locais oportunos não somente para o tratamento pontual de complicações agudas, mas também para identificar condições crônicas e realização de ações/ intervenções educativas, permitindo um controle mais abrangente e efetivo da condição de saúde e, consequentemente, dos eventos agudos e complicações ${ }^{2}$. Nos Estados Unidos, mais da metade das pessoas que buscaram os serviços de emergência demonstrou interesse em receber informações sobre HAS e DM em quiosques disponíveis nesses locais ${ }^{35}$. No contexto brasileiro, acreditase ser viável disponibilizar informações enquanto a pessoa aguarda na sala de espera, com a oferta de vídeos instrucionais e material impresso, ressaltando a importância do autocuidado e do acompanhamento na APS.

Por fim, recomenda-se a realização de estudos que investiguem o impacto das mudanças ocorridas no acompanhamento regular das pessoas com condições crônicas como a HAS e $\mathrm{DM}$, devido às medidas de distanciamento e isolamento social pela Pandemia do Covid-19. É provável que a interrupção desse acompanhamento e a limitação no acesso aos serviços da Atenção Primária tenham potencializado a recorrência dos quadros de descontrole pressórico, glicêmico e o surgimento de complicações.

\section{CONCLUSÕES E IMPLICAÇÕES PARA A PRÁTICA}

Os resultados deste estudo permitiram chegar às seguintes conclusões: a maioria das pessoas que procuraram os serviços de urgência e emergência duas ou mais vezes por complicações agudas da HAS e/ou DM era do sexo feminino, da cor de pele branca, idosa, possuindo, no máximo, nove anos de estudo, tinha companheiro ou morava com alguém, não tinha a condição de saúde cadastrada em prontuário da APS, residia em área de abrangência de UBS ainda não avaliada para certificação, porém, próximo dos serviços de pronto atendimento. Ainda, tiveram mais chances de procurar esses serviços por três ou mais vezes os usuários com menor escolaridade, sem companheiro, que buscaram apenas o pronto atendimento hospitalar ou este associado a uma das UPA municipais e por ambas as condições (HAS e DM).

Acredita-se que conhecer o perfil dos usuários que frequentam os serviços de urgência e emergência e identificar aqueles com complicações agudas da HAS e/ou DM e que desconhecem sua condição de saúde, ou que não são adequadamente acompanhadas na APS, possam subsidiar a gestão local na organização da rede de assistência e na formulação de políticas públicas e a proposição de estratégias mais efetivas para identificação, acompanhamento e busca ativa de pessoas com condições crônicas. Nesse sentido, considera-se essencial o uso sistematizado de um programa de rastreamento e/ou de prontuário único para toda a rede do município. E também que os profissionais de saúde possam valer-se da procura dos serviços de urgência e emergência para fazer orientações e sensibilizar os usuários sobre importância e vantagens de sua vinculação a uma UBS e do acompanhamento regular de sua condição de saúde nesse nível assistencial.

As limitações do estudo estão relacionadas ao uso de dados secundários sujeitos a falhas nos registros, ausência de informações e cadastros desatualizados; também à subidentificação de pessoas que procuraram esses serviços por, pelo menos, duas vezes, pois, o prontuário eletrônico em uma das UPA só foi implantado em 2019.

\section{FINANCIAMENTO}

Coordenação de Aperfeiçoamento de Pessoal de Nível Superior - Brasil (CAPES) - Código de Financiamento 001, bolsa de mestrado concedida a Patrícia Chatalov Ferreira.

\section{CONTRIBUIÇÕES DOS AUTORES}

Desenho do estudo. Patrícia Chatalov Ferreira. Sonia Silva Marcon.

Aquisição de dados. Patrícia Chatalov Ferreira.

Análise de dados e interpretação dos resultados. Patrícia Chatalov Ferreira. Elen Ferraz Teston. Rubia Mariana de Souza Santos. Verônica Francisqueti Marquete. Robson Marcelo Rossi. Sonia Silva Marcon.

Redação e revisão crítica do manuscrito. Patrícia Chatalov Ferreira. Elen Ferraz Teston. Rubia Mariana de Souza Santos. Verônica Francisqueti Marquete. Robson Marcelo Rossi. Sonia Silva Marcon.

Aprovação da versão final do artigo. Patrícia Chatalov Ferreira. Elen Ferraz Teston. Rubia Mariana de Souza Santos. Verônica Francisqueti Marquete. Robson Marcelo Rossi. Sonia Silva Marcon.

Responsabilidade por todos os aspectos do conteúdo e a integridade do artigo publicado. Patrícia Chatalov Ferreira. Elen Ferraz Teston. Rubia Mariana de Souza Santos. Verônica Francisqueti Marquete. Robson Marcelo Rossi. Sonia Silva Marcon.

\section{EDITOR ASSOCIADO.}

Candida Primo 우

\section{EDITOR CIENTÍFICO}

Marcelle Miranda da Silva (i) 


\section{REFERÊNCIAS}

1. Nilson EAF, Andrade RCS, Brito DA, Oliveira ML. Custos atribuíveis a obesidade, hipertensão e diabetes no Sistema Único de Saúde, Brasil, 2018. Rev Panam Salud Publica. 2020;44:e32. http://dx.doi. org/10.26633/RPSP.2020.32.

2. Hertz JT, Sakita FM, Manavalan P, Madut DB, Thielman NM, Mmbaga BT et al. The Burden of Hypertension and Diabetes in na Emergency Department in Northern Tanzania. Ethn Dis. 2019;29(4):559-66. http:// dx.doi.org/10.18865/ed.29.4.559.

3. Sudharsanan N, Geldsetzer P. Impact of coming demographic changes on the number of adults in need of care for Hypertension in Brazil, China, India, Indonesia, Mexico, and South Africa. Hypertension. 2019;73(4):7706. http://dx.doi.org/10.1161/HYPERTENSIONAHA.118.12337.

4. Moreira JPL, Almeida RMVR, Rocha NCS, Luiz RR. Prevalence of corrected arterial hypertension based on the self-reported prevalence estimated by the Brazilian National Health Survey. Cad Saude Publica. 2020;36(1):e00033619. http://dx. doi.org/10.1590/0102-311x00033619.

5. Marinho F, Passos VMA, Malta DC, França EB, Abreu DMX, Araújo VEM et al. Burden of disease in Brazil, 1990-2016: a systematic subnational analysis for the Global Burden of Disease Study 2016 Lancet. 2018;392(10149):760-75. http://dx.doi.org/10.1016/S01406736(18)31221-2.

6. Siqueira ASE, Siqueira-Filho AG, Land MGP. Analysis of the economic impact of cardiovascular diseases in the last five years in Brazil. Arq Bras Cardiol. 2017;109(1):39-46. http://dx.doi.org/10.5935/abc.20170068.

7. Pereira NPA, Lanza FM, Viegas SMF. Vidas em tratamento para Hipertensão Arterial Sistêmica e Diabetes Mellitus: sentimentos e comportamentos. Rev Bras Enferm. 2019;72(1):102-10. http://dx.doi. org/10.1590/0034-7167-2016-0500.

8. Gerhardt PC, Borghi AC, Fernandes CAM, Mathias TAF, Carreira L. Tendência das internações por diabetes mellitus e hipertensão arterial sistêmica em idosos. Cogitare Enferm. 2016;21(4):1-10. http://dx.doi. org/10.5380/ce.v21i4.44912.

9. Freitas PS, Matta SR, Mendes LVP, Luiza VL, Campos MR. Uso de serviços de saúde e de medicamentos por portadores de Hipertensão e Diabetes no Município do Rio de Janeiro, Brasil. Ciênc. Saúde Coletiva. 2018;23(7):2383-92. http://dx.doi.org/10.1590/141381232018237.21602016.

10. Brodeur M, Margo-Dermer E, Chouinard M-C, Hudon C. Experience of being a frequent user of primary care and emergency department services: a qualitative systematic review and thematic synthesis. BMJ Open. 2020;10(9):e033351. http://dx.doi.org/10.1136/bmjopen-2019-033351.

11. R Development Core Team. R: a language and environment for statistical computing. Vienna: R Foundation for Statistical Computing; 2020.

12. Agresti A. An introduction to categorical data analysis. 2nd ed. New York: Wiley; 2007. http://dx.doi.org/10.1002/0470114754.

13. Giolo SR. Introdução à análise de dados categóricos com aplicações. 1. ed. São Paulo: Blucher; 2017.

14. Hosmer D, Lemeshow S. Applied logistic regression. New York: John Wiley \& Sons; 2000. http://dx.doi.org/10.1002/0471722146.

15. Fawcett T. Anintroduction to ROC analysis. Pattern Recognit Lett 2006;27(8):861-74. http://dx.doi.org/10.1016/j.patrec.2005.10.010.

16. Paula GA. Modelos de regressão com apoio computacional. São Paulo: Instituto de Matemática e Estatísstica, Universidade de São Paulo;2013 [citado 2020 dez 20]. Disponível em: https://www.ime.usp.br/ giapaula/ texto_2013.pdf

17. Mello-Sampayo F. Spatial interaction healthcare accessibility model: an application to Texas. Appl. Spatial Analysis. 2018;11(4):739-51. http:// dx.doi.org/10.1007/s12061-018-9284-4.

18. Ministério da Saúde (BR), Secretaria e Vigilância em Saúde, Departamento de Análise de Situação de Saúde. Plano de ações estratégicas para o enfrentamento das doenças crônicas não transmissíveis (DCNT) no Brasil 2011-2022 [Internet]. Brasília (DF): Ministério da Saúde; 2011 [citado 3 fev 2021]. Disponível em: http://www.sbn.org.br/noticias/ acoes_estrategicas.pdf

19. Acosta AM, Lima MADS. Usuários frequentes de serviço de emergência: fatores associados e motivos de busca por atendimento. Rev Lat Am
Enfermagem. 2015;23(2):337-44. http://dx.doi.org/10.1590/01041169.0072 .2560

20. Cruz DN, Cangussu MCT, Cristino OS, Brito SS, Oliveira CB. Acesso, utilização e percepção dos usuários sobre um serviço hospitalar de emergência em Salvador, BA. Rev Ciênc Méd Biol. 2016;15(2):186-98. http://dx.doi.org/10.9771/cmbio.v15i2.15706.

21. Lima ACG, Nichiata LY, Bonfim D. Perfil dos atendimentos por condições sensíveis à Atenção Primária à Saúde em uma Unidade de Pronto Atendimento. Rev Esc Enferm USP. 2019;53:e03414. http://dx.doi org/10.1590/s1980-220x2017042103414.

22. Rêgo AS, Rissardo LK, Scolari GAS, Sanches RCN, Carreira L, Radovanovic CAT. Fatores associados ao atendimento a idosos por condições sensíveis à Atenção Primária à Saúde. Rev Bras Geriatr Gerontol. 2017;20(6):773-84. http://dx.doi.org/10.1590/198122562017020.170120

23. Radigonda B, Souza RKT, Cordoni JL, Silva AMR. Avaliação do acompanhamento de pacientes adultos com hipertensão arterial ou diabetes melito pela Estratégia Saúde da Família e identificação de fatores associados, Cambé-PR, Brasil, 2012. Epidemiol Serv Saude. 2016 mar;25(1):115-26. http://dx.doi.org/10.5123/S167949742016000100012.

24. Andrade SR, Castro RG, Senna MH. Produção das equipes da Estratégia Saúde da Família antes e após a implantação do PMAQ-AB. Cienc Cuid Saude. 2018;16(3). http://dx.doi.org/10.4025/cienccuidsaude. v16i3.33207.

25. Mendes EV. As redes de atenção à saúde. Brasília: Organização PanAmericana da Saúde, Conselho Nacional de Secretários de Saúde 2011.

26. Fontbonne A, Souza EC, Oliveira JCN, Rodrigues HM, Souza WV Cesse EÂP. Relações entre os atributos de qualidade de atenção aos usuários hipertensos e diabéticos na Estratégia Saúde da Família e o controle dos fatores prognósticos de complicações. Cad Saude Colet. 2018;26(4):418-24. http://dx.doi.org/10.1590/1414-462x201800040208.

27. Souza CL, Oliveira MV. Fatores associados ao descontrole glicêmico de diabetes mellitus em pacientes atendidos no Sistema Único de Saúde no Sudoeste da Bahia. Cad Saude Colet. 2020;28(1):153-64. http://dx.doi.org/10.1590/1414-462x202028010319.

28. Silva GF, Magalhães PSF, Silva Jr VR, Moreira TMM. Adesão ao tratamento anti-hipertensivo e ocorrência de Síndrome Metabólica. Esc Anna Nery. 2021;25(2):e20200213. http://dx.doi.org/10.1590/21779465-ean-2020-0213.

29. Wang C, Kuo HC, Cheng SF, Hung JL, Xiong JH, Tang PL. Continuity of care and multiple chronic condition simpact frequent use of out patien services. Health Informatics J. 2020 jan;26(1):318-27. http://dx.doi org/10.1177/1460458218824720.

30. Silocchi C, Junges JR. Equipes de atenção primária: dificuldades no cuidado de pessoas com doenças crônicas não transmissíveis. Trab Educ Saúde. 2017;15(2):599-615. http://dx.doi.org/10.1590/1981-7746sol00056.

31. Reis AFN, Lima JC, Beccaria LM, Ribeiro RCHM, Ribeiro DF, Cesarino CB. Tendência da morbimortalidade associada à hipertensão $e$ diabetes em município do interior paulista. Rev Lat Am Enfermagem. 2015;23(6):1157-64. http://dx.doi.org/10.1590/0104-1169.0533.2661.

32. Moura NS, Lopes BB, Teixeira JJD, Oriá MOB, Vieira NFC, Guedes MVC. Alfabetização em saúde e autocuidado em pessoas com diabetes mellitus tipo 2. Rev Bras Enferm. 2019;72(3):700-6. http://dx.doi. org/10.1590/0034-7167-2018-0291.

33. Lucena ACRM, Rêgo AS, Charlo PB, Rodrigues TFCS, Salci MA Radovanovic CAT et al. Desempenho dos serviços da atenção primária à saúde: satisfação das pessoas com hipertensão. Ciênc Cuid Saúde. 2021;20:e53086. http://dx.doi.org/10.4025/cienccuidsaude.v20i0.53086.

34. Pinto LF, Santos LJ. Prontuários eletrônicos na Atenção Primária: gestão de cadastros duplicados e contribuição para estudos epidemiológicos Ciênc Cuid Saúde. 2020;25(4):1305-12. http://dx.doi.org/10.1590/141381232020254.34132019

35. Orlando MS, Rothman RE, Woodfield A, Gauvey-Kern M, Peterson $\mathrm{S}$, Miller T et al. Public health information delivery in the emergency department: analysis of a Kiosk-based program. J Emerg Med. 2016 fev;50(2):223-7. http://dx.doi.org/10.1016/j.jemermed.2015.06.075. 